\title{
Bulas de medicamentos: compreensão pelo usuário
}

\author{
The information leaflets of medicines: understanding by the user
}

Inserción de medicamentos: comprensión del usuario

Bruno Brayan Pereira Cantareli ${ }^{1}$, Bruna Rayane Duarte Andrade ${ }^{1}$, Wellington Danilo Soares ${ }^{2}$, André Fabricio Pereira da $\mathrm{Cruz}^{3 *}$.

\section{RESUMO}

Objetivo: Avaliar a compreensão da bula de medicamento por usuários de medicamentos. Métodos: A população de estudo constituiu de 232 usuários de medicamentos, de ambos os sexos com faixa etária de 18 aos 70 anos. A coleta de dados foi realizada através de um questionário, com perguntas objetivas de múltipla escolha. Os dados coletados foram avaliados pelo programa SPSS 20.0 (Statistical Package for the Social Sciences) for Windows. Foi utilizado o teste Qui-quadrado para avaliar as diferenças de proporções dos dados categóricos sendo considerado significativo $p \leq 0,05$. Resultados: Dos entrevistados $51,7 \%$ foram do gênero masculino e $48,3 \%$ do gênero feminino e a maioria, 22,4\% tinham idade entre 50 e 59 anos de idade. $85,3 \%$ já recorreram a bula para esclarecer alguma dúvida e 38,8\% têm o hábito de consultar a bula antes de usar o medicamento. $43,5 \%$ utilizam bulário eletrônico e $66,8 \%$ entendem melhor a bula física. $34,1 \%$ não consegue entender as explicações da bula $48,7 \%$ acham a linguagem da bula muito técnica. Conclusão: Mesmo com as adequações da RDC 47, a bula ainda precisa passar por processos evolutivos afim de tornala mais direta e objetiva, com linguagens menos técnicas e letras um pouco maiores, facilitando a compreensão do consumidor.

Palavras-chave: Bula de medicamentos, Legislação sanitária, Uso de medicamentos.

\begin{abstract}
Objective: To evaluate the understanding of the drug package insert by drug users. Methods: The study population consisted of 232 drug users, of both sexes, aged 18 to 70 years. Data collection was carried out through a questionnaire, with multiple choice objective questions. The collected data were evaluated using the SPSS 20.0 program (Statistical Package for the Social Sciences) for Windows. The Chi-square test was used to assess differences in proportions of categorical data, with $p \leq 0.05$ being considered significant. Results: Of the interviewees, $51.7 \%$ were male and $48.3 \%$ female, and the majority, $22.4 \%$ were between 50 and 59 years old. $85.3 \%$ have already resorted to the package leaflet to clarify any doubts and $38.8 \%$ are in the habit of consulting the package leaflet before using the medication. $43.5 \%$ use electronic bulbs and $66.8 \%$ understand the physical package insert better. $34.1 \%$ cannot understand the explanations on the label $48.7 \%$ think the language of the label is very technical. Conclusion: Even with the adaptations of RDC 47, the package insert still needs to go through evolutionary processes in order to make it more direct and objective, with less technical languages and slightly larger letters, facilitating consumer understanding.
\end{abstract}

Keywords: Medication label, Health legislation, Drug utilization.

\footnotetext{
1 Faculdade de Saúde Ibituruna (FASI), Montes Claros - MG.

2 Universidade Estadual de Montes Claros (UNIMONTES), Montes Claros - MG.

3 Universidade Federal de Minas Gerais (UFMG), Montes Claros - MG.

*E-mail: andrefabriciocruz@yahoo.com.br
} 


\section{RESUMEN}

Objetivo: Evaluar la comprensión del prospecto de los medicamentos por parte de los consumidores de drogas. Métodos: La población de estudio estuvo conformada por 232 usuarios de drogas, de ambos sexos, de 18 a 70 años. La recolección de datos se realizó a través de un cuestionario, con preguntas objetivas de opción múltiple. Los datos recopilados se evaluaron mediante el programa SPSS 20.0 (Statistical Package for the Social Sciences) para Windows. Se utilizó la prueba de Chi-cuadrado para evaluar las diferencias en las proporciones de datos categóricos, considerándose significativo $p \leq 0,05$. Resultados: De los encuestados, el $51,7 \%$ eran hombres y el $48,3 \%$ mujeres, y la mayoría, el $22,4 \%$ tenían entre 50 y 59 años. El $85,3 \%$ ya ha recurrido al prospecto para aclarar dudas y el $38,8 \%$ tiene la costumbre de consultar el prospecto antes de utilizar el medicamento. El 43,5\% utiliza bombillas electrónicas y el $66,8 \%$ comprende mejor el prospecto físico. El 34,1\% no entiende las explicaciones del prospecto. El 48,7\% piensa que el lenguaje del prospecto es muy técnico. Conclusión: Incluso con las adaptaciones de RDC 47, el prospecto todavía necesita pasar por procesos evolutivos para hacerlo más directo y objetivo, con lenguajes menos técnicos y letras un poco más grandes, facilitando la comprensión del consumidor.

Palabras clave: Prospecto de medicación, Legislación sanitaria, Utilización de medicamentos.

\section{INTRODUÇÃO}

Todo consumidor de medicamentos, em algum momento, deparou-se com um folheto dentro da caixa contendo informações sobre ele. Esse folheto denomina-se bula e é um impresso que acompanha os medicamentos, contendo informações sobre indicação, posologia, interações medicamentosas, reações adversas, contraindicação (FUJITA PL, et al., 2014).

A bula é um documento técnico-científico que é direcionada para auxiliar tanto o usuário do medicamento, quanto o profissional da saúde. Suas informações são de suma importância por fornecer informações, sobretudo, relacionadas à composição, características e modo de uso do respectivo medicamento (FUJITA PL, et al., 2014). No Brasil, os medicamentos produzidos internamente ou importados, precisam conter a bula dentro de sua embalagem para atender as normativas vigentes (PIRES C, et al., 2015).

Item obrigatório para autorização de registros, a bula e é exigida desde 1931 como documento sanitário. E representa um importante meio de informações para o usuário sobre o respectivo medicamento (PIRES C, et al., 2015). Destaca-se que as informações contidas na bula não podem ser divergentes das informações registradas pela empresa e, por isso, ao longo do tempo, com novas exigências dos órgãos reguladores para garantir a eficácia, a qualidade e a segurança dos fármacos, os novos itens obrigatórios aumentavam e, consequentemente, também a bula (DE SOUZA JPR, et al., 2014; GUIMARÃES MAS, et al., 2017).

Dessa forma, as bulas estabelecem a principal fonte de informação sobre o medicamento, disponibilizando ao consumidor as características do fármaco contido na embalagem. As informações apresentadas são consideradas indispensáveis e são elaboradas conforme a Resolução da Diretoria Colegiada (RDC) 47, da Secretaria Nacional de Vigilância Sanitária (SNVS), de 8 de setembro de 2009, regulamentando a apresentação dos textos das bulas (AGÊNCIA NACIONAL DE VIGILÂNCIA SANITÁRIA, 2009).

As bulas representam o meio mais próximo de se recorrer às informações inerentes ao medicamento. Os pacientes, buscam, por meio delas esclarecer eventuais dúvidas. No entanto, a linguagem muito culta, técnico-científica, além das letras reduzidas, dificultam sua compreensão, principalmente, dos pacientes não especialistas em saúde. São materiais difíceis de serem compreendidos (CARMO JUNIOR NM e REIS AMM, 2017).

A Agência Nacional de Vigilância Sanitária (ANVISA) tem publicado vários protótipos de bulários e exigido a adoção destes modelos. No entanto, no Brasil, ainda se observa a ausência de uniformidade das bulas de medicamentos. E para que elas sejam úteis ao usuário é necessário que a empresa fabricante do medicamento disponibilize esse manual técnico-científico com uma linguagem clara, objetiva e atualizada em consonância com as normativas vigentes (BERVANGER EK e JÚNIOR CDAC, 2018). 
Mesmo com as adequações realizadas pela RDC 47 de 08/09/2009, pacientes e médicos, muitas vezes negligenciam a leitura da bula e sua grande importância. Existem alguns usuários que não recebem orientação de como utilizar determinado medicamento, e quando precisam pesquisar na bula, tem dificuldade de interpretar o que está escrito, prejudicando seu tratamento e saúde (AGÊNCIA NACIONAL DE VIGILÂNCIA SANITÁRIA, 2009).

A bula deve informar ao paciente os dados do fármaco em linguagem oportuna e de clara compreensão, assim como aos profissionais da saúde, com conteúdo discriminado tecnicamente em bulas isoladas, destinadas exclusivamente aos profissionais da saúde (AGÊNCIA NACIONAL DE VIGILÂNCIA SANITÁRIA, 2009; ORGANIZAÇÃO PAN-AMERICANA DA SAÚDE/ORGANIZAÇÃO MUNDIAL DA SAÚDE, 2020).

Para o usuário do medicamento, a linguagem representa uma das dificuldades de compreensão da bula. Os termos técnicos empregados pouco contribuem na comunicação com o usuário. Assim, a ausência de uma linguagem mais informal é considerada uma falha das regras normativas pelos órgãos governamentais (DE SOUZA JPR, et al., 2014; BERVANGER EK e JÚNIOR CDAC, 2018).

A linguagem técnica, descrita em letras pequenas, com espaçamento reduzido entre as linhas e as divisões em colunas presente na bula, diminui a adesão do paciente ao tratamento, já que dificulta a compreensão pelo paciente. A bula é o meio mais importante de comunicação com o paciente (TAMASINI AA, et al., 2015; RIGOTO GC, et al., 2016).

A qualidade das informações contidas nas bulas é muito importante para o paciente. E para facilitar a compreensão da bula pelo paciente, as informações devem ser claras, precisas e específicas. Elas devem apresentar uma linguagem de forma que seja de fácil leitura e compreensão pelo usuário (RIGOTTO GC, et al., 2016; DA SILVA LIMA EA, et al., 2018).

A leitura da bula é muito importante para garantir o uso racional do medicamento. Por isso, ela deve se apresentar com linguagem que facilite a compreensão por leigos. A linguagem técnica pouco contribui para o seu entendimento. $E$ uma linguagem acessível pode evitar, inclusive, agravos à saúde do paciente em função do uso inadequado do medicamento (DE QUEIROZ MJ, et al.,2012; SUSTERSIC M, et al., 2017).

Além das bulas físicas, tem-se os bulários eletrônicos que podem ser acessados através da internet. $O$ bulário eletrônico é regulamentado pelo Guia de Submissão Eletrônica de Texto de Bula e tem o objetivo de normatizar as formas de envio desses arquivos para a ANVISA e, dessa forma, assegurar o acesso às principais informações do medicamento ao usuário (AGÊNCIA NACIONAL DE VIGILÂNCIA SANITÁRIA, 2009).

Durante um determinado tratamento com terapia farmacológica é de suma importância que o paciente tenha conhecimento sobre o fármaco que está utilizando, sobre os efeitos adversos que pode haver durante o tratamento, sobre a posologia, dentre outras informações que contribuam para a adesão do paciente ao tratamento. Por isso, o objetivo deste trabalho foi avaliar a compreensão do usuário em relação a bula do medicamento.

\section{MÉTODOS}

Este estudo possui caráter descritivo, com análise quantitativa e corte transversal que objetivou avaliar a compreensão da bula por usuários de medicamentos. A amostra do estudo, foi constituída, a partir de cálculo amostral, por 232 usuários de medicamentos em uma cidade do norte de Minas Gerais - MG, de ambos os sexos, com faixa etária de 18 aos 70 anos.

A coleta de dados foi realizada através do preenchimento de um questionário, de forma individualizada, com perguntas objetivas de múltipla escolha, inerentes ao objetivo da pesquisa, sem a necessidade de identificação do voluntario. O questionário foi aplicado, somente, para aqueles que aceitaram em participar da pesquisa e assinaram o Termo de Consentimento Livre e Esclarecido (TCLE). Foram excluídos da pesquisa menores de 18 anos, indivíduos que desconheciam bula de medicamentos e aqueles que não aceitaram assinar o TCLE. O questionário, abordou três temas específicos: avaliou se o usuário consulta a bula; identificou os problemas encontrados na leitura e compreensão da bula; verificou se o profissional orientou o usuário a ler a bula. 
Os dados coletados foram reunidos, armazenados em planilha no Software Excel 2013 e avaliados estatisticamente no software IBM $\AA^{\text {SPSS }} \AA$ Statistics versão 24.0 bases de análise estatística for Windows. Foi utilizado o teste Qui-quadrado para avaliar as diferenças de proporções dos dados categóricos sendo considerado significativo $\mathrm{p} \leq 0,05$.

O projeto foi submetido ao Comitê de Ética e Pesquisa em Humanos, conforme preconizado por legislação vigente, e foi autorizado sob o parecer 3.141.479.

\section{RESULTADOS E DISCUSSÃO}

Do total de entrevistados, 120 (51,7\%) foram do gênero masculino e $112(48,3 \%)$ do gênero feminino. A faixa etária dos entrevistados foi de 18 aos 70 anos, com predomínio de indivíduos na faixa de 40 a 49 anos (22\%) e daqueles com 50 a 59 anos de idade $(22,4 \%)$. Os resultados da análise descritiva da amostra podem ser conferidos na Tabela 1.

Tabela 1 - Caracterização descritiva da amostra, $n=232$.

\begin{tabular}{cccc}
\hline Variável & N (frequência absoluta) & \% (frequência relativa) & Sig \\
\hline Gênero & & & \\
\hline Masculino & 120 & 51,7 & 0,599 \\
Feminino & 112 & 48,3 & \\
\hline Idade & & & \\
\hline 18 a 29 anos & 40 & 17,2 & 0,703 \\
30 a 39 anos & 44 & 19 & \\
40 a 49 anos & 51 & 22 & \\
50 a 59 anos & 52 & 22,4 & \\
60 a 70 anos & 45 & & 19,4 \\
\hline
\end{tabular}

Fonte: Cantareli BBP, et al., 2021.

A bula constitui um dos principais meios para o usuário esclarecer dúvidas sobre o medicamento e, por isso, é de suma importância o uso de uma linguagem que possibilite o usuário compreendê-la facilmente (RIGOTTO GC, et al., 2016). Assim, foi avaliado se o usuário de fato consulta a bula, utilizando-se as perguntas: "Alguma vez você recorreu à bula para tirar alguma dúvida gerada após uma consulta médica?" $\mathrm{E}$ "Você tem o hábito de consultar a bula do medicamento antes de fazer o uso do mesmo?". O estudo revelou que $85,3 \%$ dos entrevistados recorrem a bula quando se tem uma dúvida gerada sobre medicamento após uma consulta médica e que $38,8 \%$ consultam a bula inclusive antes de iniciar um tratamento com 0 medicamento e $32,8 \%$ dos entrevistados relataram que, às vezes, leem a bula antes de iniciarem um determinado tratamento (Tabela 2).

Tabela 2 - Avaliação do uso da bula pelo usuário, n=232.

\begin{tabular}{ccccc}
\hline Pergunta & Resposta & $\mathbf{n}$ & $\%$ & Sig \\
\hline Alguma vez você recorreu à bula para tirar alguma & $\operatorname{Sim}$ & 198 & 85,3 & \multirow{2}{*}{$0,000^{*}$} \\
dúvida gerada após uma consulta médica? & Não & 34 & 14,7 & \\
\hline \multirow{2}{*}{$\begin{array}{c}\text { Você tem o hábito de consultar a bula do } \\
\text { medicamento antes de fazer o uso do mesmo? }\end{array}$} & Não & 90 & 38,8 & \\
& Às vezes & 76 & 32,8 & \\
\hline
\end{tabular}

Fonte: Cantareli BBP, et al., 2021.

Um estudo realizado na cidade de Montes Belos - GO, mostrou que a maioria dos entrevistados não leem a bula, apesar de saberem para que ela serve, sendo somente uma minoria que não sabia para que servia. Os entrevistados que liam a bula, descreveram que a linguagem era muito técnica e culta, mostrando 
dificuldade em interpretar algumas informações (SOUSA JPR, et al., 2014). Neste estudo, no entanto, o número de usuários que recorrem a bula é estatisticamente significativo $(p \leq 0,05)$. E, por isso, é importante que as bulas tenham um desenho e linguagem adequados para facilitar a compreensão do usuário. Grande parte da população tem dúvidas sobre a indicação farmacológica, posologia, dentre outras informações sobre os medicamentos e, por isso, precisam recorrer a informações sobre eles (FERREIRA RL e JÚNIOR ATT, 2018).

Avaliou-se ainda, se os entrevistados utilizam a bula eletrônica e, além disso, por qual dos dois tipos de bula, física ou online, as dúvidas em relação aos medicamentos são esclarecidas. E, constatou-se neste estudo que $43,5 \%$ dos usuários de medicamentos entrevistados recorrem a bula eletrônica. No entanto, $66,8 \%$ compreendem melhor a bula simples (física) do que a eletrônica (Tabela 3). Muitas pessoas ainda têm dificuldade tanto de acesso, quanto de navegar na internet, o que pode estar influenciando nesses resultados.

Tabela 3 - Tipo de bula utilizado pelos usuários, $n=232$.

\begin{tabular}{ccccc}
\hline Pergunta & Resposta & $\mathbf{n}$ & $\%$ & Sig \\
\hline $\begin{array}{c}\text { Você utiliza algum bulário eletrônico, na internet ou em } \\
\text { forma de aplicativo para smartphone? }\end{array}$ & Sim & 101 & 43,5 & \multirow{2}{*}{$0,049^{*}$} \\
\hline Nual meio você consegue compreender melhor as & Bula simples & 131 & 56,5 & \\
informações? & Bula eletrônica & 77 & 36,8 & \multirow{2}{*}{$0,000^{*}$} \\
\hline
\end{tabular}

Fonte: Cantareli BBP, et al., 2021.

Neste estudo, avaliou-se também os problemas encontrados na leitura e compreensão da bula. Foi perguntado aos usuários dos medicamentos sobre a compreensão da bula. As perguntas abordadas para esse levantamento foram: "Você consegue entender as explicações contidas na bula?". E "Quando você lê a bula, as dúvidas que você tem em relação a um medicamento são tiradas pelas informações trazidas por ela?". Os dados coletados demonstraram que $65,9 \%$ dos clientes conseguem entender as explicações trazidas na bula, e que 53.9\% têm suas dúvidas esclarecidas por ela (Tabela 4).

Esse valor revela a necessidade de mais clareza e padronização nas informações dos bulários. Já que muitas dúvidas não são sanadas devido à omissão de informações relevantes, que reduz o seu valor enquanto material educativo para o usuário (VOLPATO LF, et al., 2009; CINTRA AD, 2012).

Somente na década de 70 foi percebido a importância de se utilizar uma linguagem de melhor compreensão nas bulas. Após muitos anos, durante a história da bula, melhorias foram sendo feitas (PIRES C, et al., 2015). Com a RDC 140 em 2003 surgiu uma padronização do que deveria ser obrigatório na elaboração da bula, mas somente em 2009 com a RDC 47 de 8 de setembro de 2009 foi padronizado de forma definitiva como deveria ser a elaboração, com de caracteres e tópicos obrigatórios pela lei (BRASIL, 2003).

A rotulagem brasileira, comparada com outros países, apresenta uma maior subjetividade e, consequentemente, pode gerar ambiguidade na interpretação, dificultando sua compreensão pelo usuário (CINTRA AD, 2012; MELO TAR, et al., 2020). É importante que a sua bula seja clara, completa e de fácil entendimento para, inclusive, minimizar uso irracional de fármacos, além de, eventualmente, auxiliar esclarecendo possíveis efeitos adversos que podem surgir com o uso do respectivo medicamento.

Na Austrália com o intuito de promover uma melhor compreensão das informações sobre a medicação, foi desenvolvido um método de elaboração que aborda, desde o rótulo da embalagem do medicamento até a bula. Nesse método de elaboração, todos os dados contidos são analisados para se ter uma melhor compreensão pela população. Foi criado pelo Instituto de Pesquisa em Comunicação da Austrália (CRI).

O método do $\mathrm{CRI}$, aborda todos os elementos envolvido na elaboração de rótulos para medicamentos, envolvendo o produto, legislação, design da etiqueta e teste com os usuários. Esse mesmo método foi aplicado no México por González CM (2008), em um medicamento pediátrico de venda livre, com o intuito de prover informações confiáveis e claras para os usuários, obtendo assim resultados positivos. Entretanto, para 
desenvolver uma compreensão eficiente, a bula não apenas deve ser legível, mas também deve ser compreensível, e que ao lê-la direcione o paciente para o uso correto do medicamento.

A compreensão das informações fornecidas pela bula é muito importante para contribuir na adesão do paciente ao tratamento. E a compreensão está relacionada tanto ao texto em si quanto à capacidade do usuário agir sobre informações de saúde (PIRES C, et al., 2015; FERNANDES MSJ, et al, 2016).

Tabela 4 - Compreensão da bula pelos usuários, $n=232$.

\begin{tabular}{ccccc}
\hline Pergunta & Resposta & $\mathbf{n}$ & $\%$ & Sig \\
\hline Você consegue entender as explicações contidas na bula? & Sim & 153 & 65,9 & \multirow{2}{*}{$0,000^{*}$} \\
\hline Nuando você lê a bula, as dúvidas que você tem em & Sim & 125 & 34,1 & \\
$\begin{array}{c}\text { relação a um medicamento são tiradas pelas informações } \\
\text { trazidas por ela? }\end{array}$ & Não & 107 & 46,1 & 0,237 \\
\hline
\end{tabular}

Fonte: Cantareli BBP, et al., 2021.

Com o intuito de identificar a dificuldade encontrada na leitura da bula, foi feita uma análise a respeito dos aspectos que dificultam a compreensão da bula e quais partes da bula geram maiores dúvidas. Os dados da pesquisa revelaram que $48,7 \%$ dos entrevistados relataram que a linguagem muito técnica da bula é um ponto de dificuldade, $38,8 \%$ consideram que a letra muito pequena atrapalha a leitura e 12,5\% acham que tanto a linguagem técnica como o tamanho das letras dificultam a compreensão da bula. Em relação ao campo da bula que trazem maior dificuldade de entendimento, as interações medicamentosas representam o item de maior dificuldade de compreensão $37,9 \%$, seguido pelas reações adversas $24,1 \%$ e a indicação $17,2 \%$ (Tabela 5).

Conforme os resultados, pode-se verificar que as bulas ainda precisam de adequações no tamanho da letra, da linguagem, entre outros aspectos. Uma bula com letras muito pequenas e linguagem complexa dificulta a compreensão pelo usuário leigo que, desta forma, precisa do auxílio de um profissional da área da saúde (DA SILVA LIMA EA, et al., 2018). $E$ as informações, normalmente são complexas (SUSTERSIC M, et al., 2017; FUJITA PL, et al., 2014).

Tabela 5 - Dificuldades encontradas na compreensão e leitura da bula pelos usuários, $n=232$.

\begin{tabular}{ccccc}
\hline Pergunta & Reposta & $\mathbf{n}$ & $\%$ & Sig \\
\hline & Indicação & 40 & 17,2 & \\
Onde você encontra maior dificuldade & Posologia & 19 & 8,2 & \\
de entendimento na bula? & Interações medicamentosas & 88 & 37,9 & $0,000^{*}$ \\
& Reações adversas & 56 & 24,1 & \\
& Contraindicação & 29 & 12,5 & \\
\hline \multirow{2}{*}{ Qual a dificuldade encontrada na bula? } & Letra pequena & 90 & 38,8 & \\
& Linguagem muito técnica & 113 & 48,7 & $0,000^{*}$ \\
& Ambas & 29 & 12,5 & \\
\hline
\end{tabular}

Fonte: Cantareli BBP, et al., 2021.

Em relação ao nível de dificuldade encontrado pelos usuários ao lerem a bula, somente $15,1 \%$ acham fácil compreender a bula, 55,6\% consideram o nível médio de compreensão da bula enquanto $29,3 \%$ acham difícil compreender a bula (Tabela 6). Os resultados demonstram que mesmo com as adequações da RDC 47, da Secretaria de Vigilância Sanitária de 8 de setembro de 2009 (ANVISA, 2009), a maioria dos usuários ainda consideram a bula de medicamentos de difícil compreensão $(p \leq 0,05)$. E mesmos com as adequações propostas, ainda há problemas que dificultam sua compreensão pelo usuário.

Muitas bulas ainda se encontram defasadas (DA CUNHA AM, et al., 2018). Em uma seleção de 48 bulas a partir de três farmácias de três redes, nenhuma das bulas apresentou todas as informações exigidas pelas 
normativas. E, dentre as informações apresentadas nas bulas, os cuidados de armazenamento e o prazo de validade, apresentaram-se mais frequentemente. Informações relevantes que contribuem no aumento da adesão do paciente ao tratamento são frequentemente omitidas (JUNIOR AF, et al., 2014).

Em um trabalho realizado pelo designer Gosch MC (2014), o autor demostrou que as informações contidas na bula podem apresentar um caráter mais estético; no trabalho ele reformulou as localizações dos dizeres da bula, acrescentando imagens ilustrativas de como deveria ser feito o uso da medicação, ressaltando, em um campo vermelho, as reações adversas do medicamento. Essa reformulação no designer da bula chama mais atenção do consumidor fazendo com que ele demonstre mais interesse em lê-la.

O uso irracional de medicamentos pode impactar diretamente na saúde do usuário resultando em consequências graves como, por exemplo, reações adversas, dependência e até intoxicações, caso não seja administrado corretamente. Por isso, critérios que simplifiquem e facilitem a compreensão da bula pelo usuário são indispensáveis para reduzir agravos à saúde do usuário pelo uso inadequado, ou seja, para garantir o uso racional do medicamento.

Orientar o paciente sobre a utilização correta do medicamento faz parte da conduta do médico e induz ao estreitamento do relacionamento entre o médico e o paciente, de forma que esse paciente previamente informado sobre o medicamento, tenha o poder de questionamento durante a prescrição médica. É importante também que o médico oriente o paciente a ler a bula, em caso de dúvidas após a consulta (ARRAIS PSD, et al., 2007; WEBSTER RK, et al., 2018).

Neste estudo, foi avaliado se o profissional médico orientou o paciente a ler a bula, e, para isso, foi perguntado para o entrevistado: "Seu médico te orienta a ler a bula?", e somente $24,1 \%(p \leq 0,05)$ dos usuários receberam orientação do médico para ler a bula e $76,3 \%(p \leq 0,05)$ relataram que esclarecem dúvidas sobre a bula com o profissional farmacêutico (Tabela 6 ).

Uma das principais fontes de informações sobre os medicamentos são os profissionais de saúde. Eles são considerados fontes insubstituíveis de consulta em relação aos medicamentos. Os profissionais de saúde são importantes pois conseguem relacionar o surgimento de agravos à saúde do paciente com o uso de algum fármaco (PEZATO TPJ, et al., 2015).

E diante dos resultados encontrados, é de suma importância que o profissional médico oriente o seu paciente que, em caso de dúvidas, recorra a bula, fonte oficial de informações do medicamento. Essa ação é relevante para garantir o uso racional do fármaco, garantindo a eficácia e segurança do respectivo tratamento.

O profissional farmacêutico desempenha papel informativo fundamental na instrução do paciente. Como ele atua na etapa de dispensação, torna-se indispensável no esclarecimento de dúvidas dos usuários em relação a terapia farmacológica (ZANELLA CG, et al., 2015). E, conforme observado neste estudo, apenas 23,7\% dos usuários não esclarecem eventuais dúvidas sobre a bula ou medicamento com o profissional farmacêutico. A atuação deste profissional contribui para assegurar uma segurança maior ao usuário e também evitar agravos em função do uso do medicamento.

Tabela 6 - Nível de dificuldade de compreensão e orientação de leitura da bula, $n=232$.

\begin{tabular}{ccccc}
\hline Pergunta & Resposta & $\mathbf{n}$ & $\%$ & Sig \\
\hline Qual o nível de dificuldade no & Fácil & 35 & 15,1 & \\
entendimento da leitura da bula? & Médio & 129 & 55,6 & $0,000^{*}$ \\
& Difícil & 68 & 29,3 & \\
\hline \multirow{2}{*}{ Seu médico te orienta a ler a bula? } & Sim & 56 & 24,1 & $0,000^{*}$ \\
& Não & 176 & 75,9 & \\
\hline Você tira dúvidas, sobre a bula ou & Sim & 177 & 76,3 & $0,000^{*}$ \\
medicamento com o farmacêutico? & Não & 55 & 23,7 & \\
\hline
\end{tabular}

Fonte: Cantareli BBP, et al., 2021. 
Em um estudo realizado na cidade de Montes Belos - GO por SOUZA JPR, et al., 2014, foi relatado que o percentual de pessoas que não tem o hábito de ler a bula é de $52 \%$, além disso, $81 \%$ consideram a linguagem utilizada de alta complexidade. Esses achados revelam que apesar de todo o processo evolutivo na confecção e normatização das bulas, elas ainda precisam evoluir principalmente para aproximar e facilitar a compreensão do consumidor final. Assim, se as bulas apresentassem um caráter mais dinâmico e estético elas poderiam chamar mais atenção do consumidor e melhorar a compressão deles (GOSCH MC e STROBEL NW, 2015). É necessário a atuação da ANVISA para garantir o cumprimento da legislação, para assegura o uso racional dos medicamentos e, muitas bulas não cumprem os requisitos estabelecidos (LYRA JUNIOR DP, et al., 2010).

Apesar deste estudo apresentar limitações, ele é de suma importância para aprofundar a discussão em relação a compreensão da bula pelo usuário. Apesar das normativas vigentes, são necessárias mais mudanças que aumentem a adesão do paciente a essa ferramenta que é indispensável para garantir o uso racional do medicamento.

\section{CONCLUSÃO}

Observou-se que a maior parte dos entrevistados consultam a bula e conseguem ter suas dúvidas sanadas. Entretanto, a linguagem complexa e as letras pequenas ainda são um ponto de dificuldade para a leitura delas, além disso, os médicos quase não orientam os pacientes a consultarem-na. Mas ficou evidente que os entrevistados tiram suas dúvidas em relação a medicação e bula com o farmacêutico. Como demonstrado no estudo, mesmo com as adequações da RDC 47, a bula ainda precisa passar por processos evolutivos afim de torna-se mais direta e objetiva, com linguagens menos técnicas e letras maiores, facilitando assim a compreensão. Além disso é importante que os profissionais e, principalmente, o farmacêutico oriente o paciente a ler a bula antes de usar um medicamento e aconselhe o paciente a consultar o médico ou farmacêutico, no caso de alguma dúvida em relação a bula ou medicação.

\section{REFERÊNCIAS}

1. ARRAIS PSD, et al. Aspectos dos processos de prescrição e dispensação de medicamentos na percepção do paciente: estudo de base populacional em Fortaleza, Ceará, Brasil. Cadernos de Saúde Pública, 2007; 23:927-937.

2. AGÊNCIA NACIONAL DE VIGILÂNCIA SANITÁRIA (ANVISA), 2009. Regulamentação de Bulas de Medicamentos no Brasil - RDC n 7/2009.

3. BERVANGER EK, JÚNIOR CDAC. Análise dos fatores que afetam a leitura e interpretação da bula em moradores do município de Cujubim-RO: Imagem: Cynthia Vanzella. Revista Científica da Faculdade de Educação e Meio Ambiente, 2018; 9:484-490.

4. BRASIL, Ministério da Saúde Agência Nacional de Vigilância Sanitária. Resolução №. 140, 29 de maio de 2003. Dispõe da elaboração, harmonização, atualização e publicação de bulas e medicamentos.

5. CARMO JÚNIOR NM, REIS AMM. Análise dos medicamentos relaxantes musculares de ação central comercializados no Brasil na perspectiva do Cuidado ao Idoso. Espaç. saúde (Online), 2017; p. 108-116.

6. CINTRA AD. Bulas de medicamentos alemãs e brasileiras em contraste: alguns resultados da análise linguística. Pandaemonium Germanicum, 2012; 15(20):224-261.

7. DA CUNHA AM, et al. Uma análise sobre as bulas de medicamentos no Brasil. In: Anais do XII Brazilian e-Science Workshop. SBC, 2018.

8. DA SILVA LIMA EA, et al. A importância da bula no uso responsável dos medicamentos: Imagem: Laboratório Oswaldo Cruz. Revista Científica da Faculdade de Educação e Meio Ambiente, 2018; 9:520-525.

9. DE QUEIROZ MJ, et al. Análise de bulas de medicamentos fitoterápicos comercializados em municípios do interior do Ceará, Brasil. Rev. Bras. Farm, 2012; 93(1):22-26.

10. DE SOUSA JPR, et al. O paciente e a bula e suas maiores dificuldades. Revista Eletrônica Faculdade Montes Belos, $2014 ; 7(2)$.

11. FERNANDES MSJ, et al. Incidência de Reações Adversas a Medicamentos nos Serviços de Medicina e Terapia do Hospital Central Josina Machel, em 2014." Rev Port Farmacoter 2016; 8(2): 21-27.

12. FERREIRA RL, JÚNIOR ATT. Estudo sobre a automedicação, o uso irracional de medicamentos e o papel do farmacêutico na sua prevenção: Imagem: Vida e Saúde. Revista Científica da Faculdade de Educação e Meio Ambiente, 2018; 9:570-576.

13. FUJITA PL, et al. A bula de medicamentos e a regulação de suas configurações em termos de forma e conteúdo no Brasil. Saúde soc. 2014; 23(1): 277-292.

14. GONZÁLEZ CM. Nuevas etiquetas de medicamentos para apoyar la automedicación en México: el caso de un analgésico pediátrico. salud pública de méxico, 2008; 50:453-S462. 
15. GOSCH MC, STROBEL NETO W. Estimulando o interesse e a compreensão na leitura de bulas de medicamentos através do design da informação. Anais do GAMPI Plural, 2015; 2015:46-60.

16. GUIMARÃES MAS, et al. Estratégia saúde da família e uso racional de medicamentos: o trabalho dos agentes comunitários em Palmas (TO). Trabalho, Educação e Saúde, 2017; 15(1):183-203.

17. JUNIOR AF, et al. Análise de informações técnico-científicas em bulas de medicamentos anticonvulsivantes utilizados na gravidez. Infarma-Ciências Farmacêuticas, 2014; 26(2):127-133.

18. LYRA JUNIOR DP, et al. Bulas de medicamentos usados por idosos com hipertensão: adequação da informação à regulamentação sanitária e possíveis implicações para a saúde. Ciência \& Saúde Coletiva, 2010; 15(6): $2917-2924$.

19. MELO TAR, et al. Avaliação comparativa das regulamentações, diretrizes e normas para a criação das fontes de informação oficiais sobre medicamentos (bulas) para profissionais de saúde disponibilizadas no Brasil, Europa e Estados Unidos. Brazilian Journal of Development, 2020; 6(8): 62407-62415.

20. ORGANIZAÇÃO PAN-AMERICANA DA SAÚDE/ORGANIZAÇÃO MUNDIAL DA SAÚDE (OPAS/OMS), 2020. Avaliação da assistência farmacêutica no Brasil: estrutura, processo e resultados. Disponível em <http:// https://www.paho.org/pt/brasil/ medicamentos/docs.>

21. PEZATO TPJ, CESARETTI MLR. Farmacovigilância hospitalar: importância do treinamento de profissionais na potencialização de suas ações. Revista da Faculdade de Ciências Médicas de Sorocaba, 2015; 17(3): 135-139.

22. PIRES C, et al. Legibilidade das bulas dos medicamentos: revisão sistemática. Rev Saúde Pública, 2015; 49(1): 1-13.

23. RIGOTTO GC, et al. A bula de medicamentos: a importância da leitura das bulas. Revista Científica da Faculdade de Educação e Meio Ambiente, 2016; 7(1): 16-26.

24. SOUSA JPR, et al. O paciente e a bula e suas maiores dificuldades. Rev Faculdade Montes Belos (FMB) 2014; 7(2): 10-22.

25. SUSTERSIC $M$ et al. How best to use and evaluate Patient Information Leaflets given during a consultation: a systematic review of literature reviews. Health Expectations, 2017; 20(4): 531-542.

26. TOMASINI AA et al. Prevalência e fatores da automedicação entre estudantes universitários no Norte do Paraná. Biosaúde, 2015; 17(1): 1-12.

27. VOLPATO LF, et al. Bulas de medicamentos e profissionais de saúde: ajudam ou complicam a compreensão dos usuários? Revista de Ciências Farmacêuticas Básica e Aplicada, 2009; 30(3): 309-314.

28. ZANELLA CG et al. Atuação do farmacêutico na dispensação de medicamentos em Centros de Atenção Psicossocial Adulto no município de São Paulo, SP, Brasil. Ciência \& Saúde Coletiva, 2015; 20:325-332.

29. WEBSTER RK et al. Positively framed risk information in patient information leaflets reduces side effect reporting: a double-blind randomized controlled trial. Annals of Behavioral Medicine, 2018; 52(11): 920-929. 